\title{
Complémentarités et concurrences sur le terrain du développement rural
}

L'Union fraternelle des croyants et les services déconcentrés de l'État (Dori, Burkina Faso)

\section{Koudbi Kaboré}

\section{(2) OpenEdition}

\section{Journals}

Édition électronique

URL : https://journals.openedition.org/anthropodev/682

DOI : 10.4000/anthropodev.682

ISSN : 2553-1719

Éditeur

Presses universitaires de Louvain

\section{Édition imprimée}

Date de publication : 1 décembre 2018

Pagination : 97-118

ISBN : 979-10-93476-06-3

ISSN : 2276-2019

\section{Référence électronique}

Koudbi Kaboré, «Complémentarités et concurrences sur le terrain du développement rural », Anthropologie \& développement [En ligne], 48-49 | 2018, mis en ligne le 18 juillet 2019, consulté le 21 septembre 2021. URL : http://journals.openedition.org/anthropodev/682 ; DOI : https://doi.org/ 10.4000/anthropodev.682

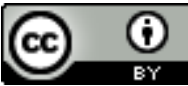

La revue Anthropologie \& développement est mise à disposition selon les termes de la Licence Creative Commons Attribution 4.0 International. 


\title{
Complémentarités et concurrences sur le terrain du développement rural
}

\section{L'Union fraternelle des croyants et les services déconcentrés de l'État (Dori, Burkina Faso)}

\author{
Koudbi Kaboré
}

Cet article analyse l'évolution des relations entre les services techniques de l'agriculture et les acteurs de l'Union fraternelle des croyants (UFC) sur le terrain du développement rural à Dori au nord du Burkina Faso, depuis la création de l'UFC en 1973, au moment de la sécheresse. Alors que l'UFC s'était imposée comme un acteur central dans les années 1970 et 1980, et était perçue comme contribuant aux politiques de l'État, et que cette collaboration continue à être mise en avant par l'administration territoriale, la réforme du secteur agricole de 2001 a rebattu les cartes. En renforçant la volonté de contrôle étatique sur les organisations paysannes locales, et en faisant des services techniques des prestataires de projets, la réforme attise la rivalité entre ces deux acteurs du développement rural quant à la supervision des organisations paysannes et au contrôle des moyens financiers. Les agents des services techniques de l'agriculture contestent la bienveillance de l'administration publique envers I'UFC qui, selon eux, lui permet de développer des pratiques hors normes et de se soustraire à leur contrôle.

The current article is an analysis of the evolution of the relationships between the technical services of agriculture and the actors of 'Union Fraternelle des Croyants' (UFC) concerning the rural development in Dori, northern Burkina Faso, since the creation of UFC in 1973 during the droughts. Whereas UFC was endeavouring like a foremost actor in the 1970s and 1980s, and was considered to be a contributor to State policies, and that this collaboration continues to being forwarded by the territorial administration, 2001 reform of agricultural sector has given way. By reinforcing the will of State control over local peasants' organisations, and using technical services like projects providers, the reform has brought about fierce rivalry between those two actors of rural development concerning 
the supervision of peasants' organisations and the control of financial means. The workers of the technical services of agriculture are distrusting the kindness of the public administration to UFC, which according to them, permit it to develop out of norms practices and to escape their control.

\section{Introduction ${ }^{1}$}

Créée en 1973 lors de la grave sécheresse qu'a connue le Burkina Faso (alors Haute-Volta), I'Union fraternelle des croyants (UFC) joue depuis ce temps un rôle central dans le développement rural dans la région de Dori, autour d'une double originalité : d'une part, cette ONG est codirigée par des représentants musulmans et catholiques et promeut le dialogue interreligieux, d'autre part, les financements et les appuis dont elle a bénéficié depuis cette date en ont fait la principale organisation locale de développement agricole et rural de la zone. Bien qu'agissant de façon autonome des pouvoirs publics, ses interventions s'inscrivaient dans les priorités politiques et étaient appréciées des pouvoirs publics.

L'année 1999 a cependant doublement marqué une recomposition dans les relations entre I'UFC et l'État (gouvernement, administration territoriale et services techniques déconcentrés) et accru les concurrences et rivalités avec les services techniques. D'une part, l'organisation s'est relancée après une longue crise d'une douzaine d'années. Afin de se repositionner dans le paysage associatif de la région et de démontrer sa volonté de collaboration avec l'État burkinabè, I'UFC invita le hautcommissaire de la province du Séno à présider son assemblée générale de $1999^{2}$ - la toute première en 30 ans d'existence! -, qui marquait la renaissance de l'organisation. Le besoin d'un soutien permanent des autorités administratives a ensuite poussé les dirigeants de l'UFC à pérenniser une pratique qui se voulait au départ circonstancielle : depuis

${ }^{1}$ Ce texte présenté à la $6^{e}$ session de l'université d'été du LASDEL (Niamey, 15-28 septembre 2014) a été discuté à l'atelier d'écriture scientifique de I'APAD tenu à Cotonou du 26 au 29 septembre 2016. Je remercie particulièrement Philippe Lavigne Delville pour ses nombreuses contributions.

2 Union fraternelle des croyants, mémoire de la première assemblée générale, Dori, 4 et 5 mars 1999. 
1999, c'est devenu une habitude pour eux - comme pour de nombreux projets ou ONG - de faire présider ou parrainer leurs manifestations par les autorités administratives ou ministérielles.

D’autre part, l'État a au même moment redéfini sa politique de développement rural et d'appui aux organisations rurales. Alors qu'il avait depuis les années 1970 largement laissé les organisations paysannes entre les mains des ONG et des associations de développement, il réaffirme une volonté de cohérence et de contrôle, en créant les chambres régionales d'agriculture (CRA) chargées d'exercer tutelle et appui aux organisations locales.

La $17^{\mathrm{e}}$ assemblée générale qui s'est tenue le jeudi 26 février 2015 a été présidée par le secrétaire général de la région, Ambroise Somé. Dans son discours, il a relevé une parfaite collaboration entre I'UFC et son administration. Ce discours officiel est contredit sur le terrain par les agents des services techniques de l'agriculture qui évoquent des tensions entre eux et les acteurs de I'UFC. Le non-respect des normes étatiques dans l'encadrement des organisations paysannes et dans les réalisations hydrauliques faites par I'UFC, ainsi que des griefs d'incompétence sont les éléments sur lesquels se cristallisent les tensions. Les agents des services techniques déconcentrés de l'État contestent la bienveillance affichée par l'autorité administrative envers l'ONG confessionnelle, qui lui permet selon eux de développer des pratiques hors normes et de se soustraire à leur contrôle.

Cet article analyse les tensions qui opposent les services techniques de l'agriculture aux acteurs de l'UFC sur le terrain du développement rural à Dori. Il interroge les enjeux des rivalités sourdes qui se développent entre ces deux acteurs de la sécurité alimentaire et du développement rural, dans une apparente indifférence des autorités administratives et ministérielles de tutelle.

Le discours des agents des services techniques déconcentrés de l'État s'enracine dans une logique de professionnalisation, qui appelle les ONG et associations du domaine du développement rural à s'adapter aux règles fixées par l'État. Jean Freyss, qui souligne l'ambiguïté du concept, propose de distinguer la professionnalisation "dans» les ONG et la professionnalisation « des » ONG (2004: 758). La première se concrétise 
par la mobilisation, au sein de l'ONG, des compétences techniques jugées nécessaires à l'efficacité d'une action (des savoirs techniques spécialisés, et des techniques de gestion de projet en particulier) :

"La professionnalisation signifie que l'ONG mobilise, dans chaque domaine utile pour son activité, des personnes disposant des compétences reconnues (par l'expérience acquise mais de plus en plus par des diplômes) comme constitutives d'un métier nommément désigné » (ibid. : 762).

La seconde renvoie à la capacité de l'ONG à s'ancrer dans des espaces et à inscrire ses actions dans des dynamiques de changement social et à accroître ainsi la pertinence et l'efficacité de ses actions, par rapport aux acteurs à qui elles sont destinées. La professionnalisation " dans " les ONG, qui privilégie les pratiques normées et l'atteinte de résultats contractuels, peut être contradictoire avec la recherche d'impacts durables.

L'analyse présentée ici repose sur des entretiens et des observations réalisés à Dori et à Ouagadougou de 2014 à 2015 auprès des coordonnateurs de I'UFC (actuel et anciens) et des responsables des services techniques de l'agriculture (la chambre régionale d'agriculture (CRA) et la direction régionale de l'agriculture, de l'hydraulique et de la sécurité alimentaire (DRAHSA)) du Sahel. Avec les coordonnateurs de I'UFC, nous nous sommes intéressés à la structuration de l'UFC, ses rapports avec ses partenaires (donateurs et bailleurs de fonds, y compris les structures étatiques), sa culture organisationnelle et ses registres de compétences. Avec les responsables des structures étatiques, nous avons discuté des questions de compétences, des mécanismes de collaboration entre les acteurs de la sécurité alimentaire, des enjeux de la professionnalisation et des récentes réformes dans le secteur de l'agriculture. À Dori, les acteurs de I'UFC nous ont permis d'observer différents moments de la vie de leur organisation (assemblées générales, manifestations publiques notamment les éditions 2014 et 2015 des Journées du maraîcher du Sahel (JMS)). Les situations observées ont permis de décrypter la mise en scène de la collaboration cordiale au sommet et les tensions à la base autour de la distribution des rôles, de la définition des priorités, et du choix des partenaires. Ces observations ont informé et orienté les situations d'entretiens, et vice versa. L'étude a également intégré des données d'archives. En raison d'une dispersion et d'une mauvaise conservation de 
leurs archives, les acteurs rencontrés manquaient d'informations sur les activités et les rapports de l'UFC avec les pouvoirs publics pour la période comprise entre 1969 et 1999, ce que l'exploitation des archives de l'ancienne préfecture de Dori (actuel gouvernorat du Sahel) a permis de comprendre.

Dans la première et la deuxième partie, nous abordons dans une perspective historique le contexte de la création de I'UFC, et l'implication de ses acteurs dans la politique de développement rural de l'État burkinabè (1969-1999). Durant cette période, I'ONG a évolué de l'humanitaire vers le développement rural intégré en lien avec les orientations de l'État. La troisième partie analyse les réformes de 1999 et de 2001 sur le secteur agricole et les évolutions qu'elles ont induites dans les relations entre les acteurs de l'UFC et les agents techniques du ministère de l'Agriculture. L'accent est mis sur les différences de traitement de I'UFC par les autorités administratives et les agents des services techniques de l'agriculture sur la mise en œuvre de la politique de développement rural.

\section{L'humanitaire catholique et les débuts de l'UFC}

Depuis le début des années 1900, le champ d'action des missionnaires catholiques pour la promotion de l'homme en Haute-Volta a été vaste. Parfois imposées par le contexte social, parfois par le besoin de consolider l'évangélisation, les options préférentielles des missionnaires allaient de la lutte pour la libération de la femme, à la lutte contre la pauvreté en passant par la promotion de la santé et de l'éducation. Dans un Sahel burkinabè aride et exposé aux famines, l'église catholique à Dori s'orienta vers I'humanitaire dès sa fondation en 1961. Comme dans nombre d'églises, l'humanitaire catholique prit appui sur le Secours catholique, l'association caritative de l'Église. L'aide qu'il apporte aux pauvres trouve son fondement dans l'enseignement social de l'Église, et ne comporte pas d'objectifs de conversion des populations non chrétiennes. Mais en 
contexte musulman ${ }^{3}$, l'aide catholique, et plus globalement l'aide chrétienne, ne peut être considérée par les musulmans comme un altruisme dépourvu d'intention apologétique. S'étant représenté l'aide du Secours catholique comme entrant dans une stratégie d'évangélisation, les musulmans manifestèrent de la prudence à en recevoir. Rejetée au départ, l'aide catholique se révéla pourtant importante dans la lutte contre la faim dans la région de Dori et de Gorom-Gorom lors de la grave sécheresse de 1969-1973 qui a durement touché le Sahel burkinabè (Gado, 1993 ; Janin, 2008 ; Piveteau, 1994 ; Enée, 2010).

La sécheresse a frappé une région dont les structures économiques et I'intégration à l'État voltaïque au sortir de la colonisation étaient encore faibles. Première crise humanitaire postcoloniale, le défi qui s'est présenté aux pouvoirs publics était celui de l'organisation et de l'acheminement de l'aide dans d'une région où le réseau routier et les moyens de transport et de stockage à tous les niveaux étaient insuffisants pour atteindre des villages isolés ${ }^{4}$. L'évaluation de la catastrophe et des besoins alimentaires des populations a donc retardé le déploiement du dispositif public de l'aide $^{5}$, laissant le terrain aux ONG et associations humanitaires (Guignard, 1996 ; Poussart-Vanier, 2005). Le Secours catholique paroissial fut le relais local de la plupart des ONG humanitaires internationales ${ }^{6}$ pour la coordination et la distribution de l'aide d'urgence. II construisit une légitimité sur la transparence et la bonne gestion de l'aide, qui lui valut une place privilégiée dans le champ de l'humanitaire et la considération de la communauté musulmane de Dori. Fort de cette confiance retrouvée, le Secours catholique put s'adjoindre des collaborateurs musulmans. Un comité mixte islamo-catholique, mis en place pour renforcer l'équipe de bénévoles du Secours catholique, et qui prit la dénomination d'« Union

\footnotetext{
${ }^{3}$ Le Sahel est une région de culture musulmane. L'islam imprègne la vie de plus de $96 \%$ de sa population (qui est musulmane selon le recensement général de la population de 2006).

${ }^{4}$ Rapport du CILSS sur la situation alimentaire et nutritionnelle dans le Sahel affecté par la sécheresse, Rome, 28 août 1973, Archives nationales du Burkina Faso (ANBF), 9V324, p. 28.

${ }^{5}$ Rapport du CILSS sur la situation alimentaire et nutritionnelle dans le Sahel affecté par la sécheresse, op. cit.

${ }^{6}$ Caritas internationalis, Cathwel (aujourd'hui Catholic relief service), le Comité catholique contre la faim et pour le développement (CCFD), Oxfam-Afrique faisaient partie de ces ONG.
} 
fraternelle des croyants " à la fin de l'année 1969, finit par se substituer au Secours catholique (Kaboré, 2016).

Parallèlement, l'extension des actions de I'UFC dans l'Oudalan au nord de Dori fit naître l'Association d'entraide fraternelle (AEF), un regroupement de musulmans et de catholiques à l'image de I'UFC-Dori. L'AEF, dont le but se limitait à la distribution de l'aide d'urgence, devint en 1972 la branche UFC de Gorom-Gorom. En 1973, les missionnaires catholiques et les autorités musulmanes dans les deux villes répondirent au désir de certaines agences de l'aide internationale de voir se maintenir la dynamique interreligieuse par l'institutionnalisation des deux associations de solidarité. Elles sont reconnues officiellement en 1973 comme ONG humanitaires et de développement dans la région de Dori et de Gorom-Gorom?. Les deux ONG « sœurs " ont été aussitôt mises sous la seule tutelle de l'Église catholique et de ses organismes caritatifs qu'étaient la Caritas voltaïque et le Bureau d'études et de liaison (BEL) en vue de la réflexion et du financement de leurs activités ${ }^{8}$. L'église catholique de Dori en était le répondant juridique, tandis que le rôle de la Caritas voltaïque et du $\mathrm{BEL}^{9}$ était surtout de faciliter les relations avec les organismes missionnaires et les ONG internationales ${ }^{10}$. Cette époque qui coïncide avec l'engagement des missions chrétiennes pour le développement a vu s'amorcer un transfert de générosité missionnaire dans le Tiers Monde (Prudhomme, 2005), dont les UFC ont été largement bénéficiaires. Jusqu'à la fin de l'année 1980, la coopération missionnaire leur fournissait argent, matériel et volontaires. Jacques Guignard ${ }^{11}$ qui fut

\footnotetext{
7 Statuts de I'UFC, 6 décembre 1974, Archives du gouvernorat du Sahel/Dori.

${ }^{8}$ Statuts de l'UFC, 6 décembre 1974, op.cit.

9 Le Bureau d'études et de liaison (BEL) est cette structure créée en 1973 par l'église catholique de Haute-Volta pour traduire en acte concret sa vision du développement. Son credo, c'est impliquer fortement les populations dans l'identification des priorités, la prise des décisions ainsi que dans la réalisation des activités, de sorte qu'elles collaborent avec les animateurs dans la recherche des solutions aux problèmes qui les concernent.

${ }^{10}$ Amadou Gourovo Sidibe et André Marty, 1980, rapport d'évaluation des activités de I'Union fraternelle des Croyants (UFC) de Dori (Haute-Volta), Dori, archives du gouvernorat du Sahel/Dori, p. 11.

${ }^{11}$ Ingénieur, il fut l'un des nombreux laïcs en Europe qui, au nom de leurs églises, étaient venus se solidariser avec les missionnaires et les populations dans la région de Dori et de Gorom-Gorom.
} 
un des volontaires pour le compte de l'UFC de Gorom-Gorom relate dans son ouvrage Le Sahel de la sécheresse : septembre 1972 à septembre 1974, comment à partir de 1975, sans abandonner le terrain de l'humanitaire, les UFC orientèrent leurs actions vers des projets durables (reboisement, construction d'ouvrages hydrauliques) dans le cadre de la politique de développement rural de l'État, où elles s'étaient imposées comme un acteur central dans les années 1970 et 1980. Les pages qui suivent sont consacrées seulement à l'action de I'UFC-Dori, couvrant territorialement les provinces du Séno et du Yagha selon le découpage administratif actuel.

L'UFC dans la politique de développement rural de l'État (19751999)

Les ONG, surtout confessionnelles, revendiquent une indépendance vis-àvis des pouvoirs publics. En dépit de cette indépendance revendiquée, elles sont comme les ONG laïques, souvent soumises à des contraintes et des exigences de fonctionnement qui les rapprochent nécessairement des autorités publiques (Diawara, 1999; Kaag et Saint-Lary, 2011 ; Leblanc et al., 2013). Le positionnement vis-à-vis de l'État pouvant se révéler un aspect important de leur évolution, les acteurs de I'UFC ont dès 1974 affirmé leur volonté de collaborer avec l'administration locale dans la mise en œuvre de la politique alimentaire et de développement rural du gouvernement voltaïque ${ }^{12}$. Cette collaboration s'est traduite par I'intégration des interventions de I'UFC dans les programmes des deux structures permanentes de l'État, les offices céréaliers nationaux (OFNACER) et les organismes régionaux de développement $(\mathrm{ORD})^{13}$. À I'instar d'autres acteurs non étatiques, I'UFC a aligné ses activités sur les priorités nationales et adhéré en 1975 au Secrétariat permanent des ONG (SPONG).

\footnotetext{
${ }^{12}$ Statuts de l'UFC, 6 décembre 1974, op. cit.

${ }^{13}$ Les OFNACER étaient des établissements publics à caractère commercial qui œuvraient à assurer la disponibilité des céréales et à stabiliser les prix. Ils travaillaient en collaboration avec les ORD, principaux relais des politiques agricoles dans les régions. Créés en 1966, les ORD avaient en charge la vulgarisation de thèmes techniques, l'encadrement des paysans, la collecte de données statistiques pour la constitution des bilans céréaliers et maraîchers.
} 
L'inscription de l'UFC au SPONG et à l'ORD lui a conféré le statut d'ONG auxiliaire de l'État à Dori et à Gorom-Gorom. La participation des acteurs de I'UFC à la politique publique alimentaire et de développement rural devait prendre une forme indirecte. Elle conservait la liberté de mobiliser des ressources matérielles et financières au profit de ses projets propres, mais elle devait toutefois en confier la supervision aux structures étatiques. Dans les faits, l'UFC a constitué le rouage essentiel du dispositif étatique. Devant le constat que les capacités matérielles et financières de I'ORD du Sahel étaient limitées par rapport à l'ampleur des besoins, les différents administrateurs de la préfecture de Dori ont vivement encouragé et soutenu une synergie des compétences et des moyens entre I'ORD et l'UFC. L'ORD tirait de cette synergie d'action des capitaux et du matériel de I'UFC. L'appui de I'UFC à I'ORD revêtait deux formes. La première était le prêt de logistique, procédé par lequel l'UFC prêtait ses camions, ses machines Caterpillar, ses foreuses et ses compresseurs aux projets routiers et d'infrastructures de l'ORD. La deuxième était le financement ou la prise en charge de projets étatiques. À la demande des autorités locales, I'UFC recherchait le financement et exécutait des projets qui étaient du ressort de l'ORD. Elle a ainsi financé et réalisé divers projets étatiques dont le reboisement, la construction de centres de formation agricole, magasins de stockage de vivres, forages, etc. Les archives administratives ont gardé des traces de cette collaboration de I'ORD avec I'UFC. En 1978 par exemple, le préfet du département du Sahel relevait que :

"L'Union fraternelle des croyants (UFC) basée à Dori et à GoromGorom mérite de toutes ces associations une mention très spéciale. [...] Elle a construit le centre de perfectionnement rural (CPR) de Diomga (sous-préfecture de Dori) qui a pour rôle l'éducation des élèves et des agriculteurs en matière de vulgarisation dans le Sahel14 "

En 1979, il rendait encore compte au gouvernement de ce que la poursuite par I'UFC de son " programme de forage et de creusement de

${ }^{14}$ Rapport politique du département du Sahel, année 1978, ANBF, 8V291. 
puits, de surcreusement des mares " dans l'Oudalan a permis le retour de beaucoup d'éleveurs nomades qui avaient déserté leurs campements ${ }^{15}$.

En retour, I'ORD mettait à la disposition de I'UFC ses agents pour renforcer son équipe technique dans la réalisation et la supervision de ses projets propres. L'équipe permanente de I'UFC à l'époque se limitait à 12 personnes ${ }^{16}$ que venaient renforcer des volontaires de la coopération missionnaire. Les techniciens de l'ORD représentaient une ressource de proximité, capables de suppléer à l'absence des techniciens expatriés.

Le régime révolutionnaire (1983-1987) adopta des mesures qui poussèrent encore les ONG à une collaboration active avec les services étatiques. Le statut d'ONG était subordonné à l'approbation du Bureau de suivi des ONG (BSONG) ${ }^{17}$, sur la base de critères de structuration, de fonctionnement interne, de qualité du personnel, de compétences reconnues dans des domaines particuliers, de degré d'implantation des ONG (Tallet, 1989 : 40 ; Piveteau, $1994: 3-5$ ). Si les deux derniers critères ont conféré un positionnement central pour I'UFC dans le domaine de I'hydraulique rurale dans le paysage des ONG au Sahel, les exigences de structuration, de fonctionnement et de qualification professionnelle devaient la pousser vers la professionnalisation " dans » les ONG.

Le processus de professionnalisation amorcé sous la Révolution avec le recrutement d'un personnel de gestion (un chargé de projets et un trésorier), et d'un personnel ouvrier au sein du personnel interne de I'ONG, s'est accéléré à partir de 1999 sous la pression de certains des donateurs dont Misereor. II s'est traduit à cette période par une profonde restructuration, le recrutement de cadres techniques et l'accroissement

\footnotetext{
${ }^{15}$ Rapport du premier trimestre du département du Sahel, 1979, ANBF, 8V291.

${ }^{16}$ Elles formaient le bureau de l'UFC, à raison de six personnes par communauté religieuse membre et un trésorier affecté par l'église suivant les clauses de la tutelle. L'Église catholique et la communauté musulmane en sont les communautés membres qui fournissent à l'UFC des délégués pour constituer son bureau et soutenir ses activités.

${ }^{17}$ Créé en mai 1984 en remplacement du Secrétariat permanent des ONG (SPONG), le BSONG se définit comme une structure destinée à renforcer les capacités des ONG en vue de les rendre aptes à participer efficacement à la mise en œuvre de la politique agricole de la Révolution. Sa spécificité par rapport au SPONG était d'apparaître aux yeux des ONG comme une structure d'accompagnement et non de contrôle.
} 
des services. La restructuration a consisté à une séparation des rôles entre les agents techniques et de gestion recrutés sur la base de leurs compétences et l'ensemble des bénévoles locaux. La restructuration visait l'efficacité et l'amélioration de la gouvernance de l'avis de cet ancien gestionnaire :

"C'était le bureau qui était à la fois instance de délibération et équipe d'exécution. Il n'y avait pas de services bien définis, ni de rôle clairement attribué à chacun des membres du bureau en dehors de la trésorerie. Ce mode de fonctionnement était source de confusion et de malversation. C'était du moins ressenti ainsi » (Ouagadougou, 27 février 2015).

Le personnel permanent était bien limité, mais le budget de I'UFC prenait en compte le groupe de bénévoles locaux qui participaient à l'animation des activités. Misereor, à la suite d'autres donateurs, a décidé de ne plus prendre en compte ces derniers dans le financement de l'UFC. Revendiquant la " paternité » de l'ONG, l'évêque ${ }^{18}$ et le grand imam de Dori, respectivement conseiller juridique et conseiller moral de I'UFC, militèrent pour leur maintien comme membres à part entière au sein de l'ONG. Sur la base de ce compromis, les délégués des deux communautés adoptèrent lors de l'assemblée générale de mars 1999 un organigramme à deux composantes: une composante religieuse chargée de la promotion du dialogue interreligieux, et une composante technique pour le volet développement.

La composante religieuse comprend les membres fondateurs (Église catholique et communauté musulmane) et les représentants des coopératives agricoles de I'UFC dans les campagnes partant du principe admis à l'origine que tout catholique et tout musulman sont membres de I'UFC. Toutefois, des délégués dûment mandatés par les communautés religieuses membres et les coopératives agricoles siègent à l'assemblée générale de I'UFC. L'équipe technique est organisée en trois pôles de services : le service de coordination des projets et programmes, celui de la

${ }^{18}$ Du diocèse de Fada N'Gourma dont dépendait la paroisse de Dori avant son érection en diocèse en 2006. 
comptabilité, et celui du suivi-évaluation ${ }^{19}$. Chacun de ces pôles de services intègre des départements, qui sont des sous-unités de coordination des projets. Un gestionnaire pilote l'équipe composée de 25 salariés aux profils variés. Parmi eux figurent des cadres moyens (ouvriers, techniciens, comptables, secrétaires) détenteurs pour la plupart de diplômes d'études secondaires (CAP en bâtiment ou mécanique, BEP en comptabilité et BEPC) et des cadres supérieurs (les chefs des pôles de services et des départements) détenteurs de diplômes universitaires (maîtrise et master en études de développement d'universités européennes).

La mise en place d'instances (assemblée générale, conseil d'administration, bureau du conseil d'administration) achève la restructuration de I'UFC. À des niveaux variables, toutes ces instances sont délibératives et fonctionnent sur le principe de la parité de membres et de la présidence tournante entre l'Église catholique et la communauté musulmane. Chaque communauté religieuse est représentée à l'assemblée générale par 24 membres. Le principe est le même pour la composition du conseil d'administration qui compte 24 membres, et le bureau du conseil d'administration où siègent 12 membres. L'évêque et le grand imam sont membres de droit de l'assemblée générale et du conseil d'administration. La vie de l'ONG et les grandes orientations de son action sont débattues lors de l'assemblée générale ordinaire (une fois l'an) et des sessions du conseil d'administration qui se tiennent ordinairement une fois par mois.

Il faut souligner que le seul membre de l'équipe technique qui participe à ces instances est le gestionnaire. II rend compte à l'assemblée générale et au conseil d'administration de l'exécution des projets, de la gestion des ressources, des relations extérieures, etc. Bien qu'étant la cheville ouvrière de l'ONG, le gestionnaire a des prérogatives très limitées. Le pouvoir de décision revient en réalité à la composante religieuse de l'UFC.

L'UFC réalise des projets parallèles en vue de la prise en charge des bénévoles qui la composent. En plus des petits projets socioéconomiques ${ }^{20}$ que l'ONG obtient souvent sur le marché de l'aide publique nationale,

${ }^{19}$ Le nombre de services n'est pas limitatif. II s'accroît ou diminue en fonction des nouvelles contraintes. Le service de la communication s'est ajouté aux trois autres en 2010.

${ }^{20}$ Appelés projets locaux en opposition aux projets structuraux que financent les donateurs. 
formations, conférences, sensibilisations sur le dialogue interreligieux et la culture de la paix sont également des projets qui attirent de plus en plus de subventions pour la partie religieuse de I'UFC (Langewiesche, 2011; Kaboré, 2017).

La réorganisation actée par l'assemblée générale de 1999 a entraîné deux conséquences majeures pour I'UFC. La première fut la professionnalisation de l'équipe technique, qui est passée par sa salarisation avec en corollaire une compression du personnel ${ }^{21}$ et des besoins accrus de ressources régulières pour financer les salaires. La seconde, qui découle de la première, fut une concurrence accrue avec les autres intervenants du domaine de la sécurité alimentaire et du développement rural : à partir de 1999, dans un contexte marqué par le retour de l'État dans le champ du développement, la légitimité de I'UFC se reconstruit sur une logique de compétence technique, fondée sur des acquis dans l'hydraulique et l'organisation des paysans.

Les réformes du secteur agricole dans les années 2000 : des tensions entre l'UFC et les services étatiques

L'échec des programmes d'ajustement structurel à la fin des années 1990 et la critique des interventions externes ont conduit les bailleurs de fonds à replacer l'État au centre des politiques publiques. À partir des années 1990 et des politiques de réduction de la pauvreté, et plus encore avec la déclaration de Paris sur l'aide au développement (Lavigne Delville, 2013), les bailleurs de fonds affirment s'aligner sur les politiques nationales. Cette relégitimation de l'État a permis à ce dernier d'accroître sa tutelle sur les organisations privées de développement. Au Burkina Faso, les réformes dans les domaines de la sécurité alimentaire et du développement rural de 1999 et de 2001 s'inscrivent dans cette perspective. Elles ne répondent pas seulement à un souci de régulation des interventions des structures et

\footnotetext{
${ }^{21}$ La mesure a concerné certains membres du personnel de gestion et ouvrier du groupe de bénévoles de l'UFC, dont on pense que les profils ne correspondaient plus aux emplois qu'ils occupaient, ou que le recrutement n'a pas suivi les règles. Après leur indemnisation, les puisatiers par exemple qui étaient toujours sollicités par I'UFC y demeurèrent quelques années avant de se regrouper en association autonome.
} 
organismes d'appui dans le monde rural, il s'agit aussi pour l'autorité publique d'être arbitre du jeu en fixant les règles, et de reprendre le contrôle de secteurs stratégiques.

Par la loi n 014-1999 sur les chambres régionales d'agriculture (CRA), l'État incite les acteurs de la sécurité alimentaire et du développement rural à susciter la création de regroupements et de fédérations d'organisations locales ${ }^{22}$. Les CRA sont des établissements publics de l'État à caractère professionnel. Elles sont placées sous une double tutelle, technique du ministre de l'Agriculture et financière du ministre des Finances. Elles bénéficient d'une autonomie de gestion et de la personnalité morale, ce qui les distingue des services techniques déconcentrés de l'État. Les CRA ne disposant que de faibles ressources structurelles publiques, elles sont autorisées à réaliser des prestations de services pour s'autofinancer. Elles cherchent dès lors à se positionner comme prestataires pour des projets.

La direction régionale de l'agriculture, de l'hydraulique et de la sécurité alimentaire (DRAHSA) n'est quant à elle pas censée faire de prestations mais, dans les faits et face aux contraintes de ressources et à la faiblesse des moyens de fonctionnement, ses agents, officiellement, réalisent aussi des prestations au service des projets et des ONG, et en tirent des compléments de revenus. Cette marchandisation de fait de certains services de la DRAHSA explique en partie les critiques que ces agents portent sur I'UFC. Car ce qui se joue dans les tensions entre les structures relevant de la tutelle étatique et les ONG d'appui est le contrôle des organisations paysannes. Comme le montre l'anthropologie du développement (Barbee, 1987; Olivier de Sardan et Bierschenk, 1993 ; Blundo, 1995), lorsque les agents publics de l'État deviennent des courtiers qui négocient entre l'État et les communautés, ils entrent en conflit avec les autres acteurs de leur domaine de compétence.

Dans le développement rural, les actions de l'UFC reposent essentiellement sur l'hydraulique villageoise et agropastorale. Elle réalise des

\footnotetext{
${ }^{22}$ Les acteurs d'une même filière peuvent se constituer selon quatre formes fédératives : les coopératives, les groupements, les unions de coopératives ou de groupements, les fédérations ou confédérations de coopératives ou de groupements.
} 
micro-barrages, aménage des périmètres et encadre des coopératives pour la culture maraîchère. Conformément à la loi $n^{\circ} 014$, ses acteurs collaborent à des degrés variables avec les agents de la CRA et de la DRAHSA. Or, les cadres techniques de référence que les agents techniques de l'État imposent aux ONG et associations ne rencontrent pas souvent l'adhésion de l'UFC. Ce sont ces réticences qui alimentent particulièrement les mésententes entre ses acteurs et les agents des services techniques de l'État.

L'article 6 de la loi $n^{\circ} 014$ stipule que les CRA sont consultées pour toutes questions se rapportant à la définition et la mise en œuvre des politiques et programmes d'appui aux agriculteurs ${ }^{23}$. Un des responsables de la CRA du Sahel explique que c'est conformément à cette disposition qu'ils appellent les associations et ONG membres à créer des coopératives et des unions faîtières. Elles sont financées par la CRA pour mettre en place les fédérations associatives, qu'elles délèguent ensuite à la CRA pour l'encadrement (Dori, 4 août 2015).

La loi est incitative. Or, les agents de la CRA en font un impératif de leur collaboration avec les organismes d'intervention. Leurs appels aux ONG qui ne s'y conforment pas prennent des allures de reproches souvent sévères. C'est ce qui ressort des propos de ce responsable du service des ONG et organisations paysannes à l'endroit des dirigeants de l'UFC :

"Non seulement ils ne veulent pas respecter le schéma qui est qu'il faut mettre en place des unions faîtières, mais en plus ils veulent des unions à eux. Ils parlent d'unions des maraîchers. Cela n'existe pas dans le schéma. Normalement on ne peut pas les financer. Deuxièmement, les unions restent leurs unions, c'est-à-dire qu'il n'y a pas de possibilité d'associer d'autres producteurs à ceux qui forment leurs groupements si bien que vous ne pouvez pas travailler directement avec ces unions-là. L'UFC refuse le schéma parce qu'elle veut toujours avoir un contrôle sur les groupements qu'elle met en place » (Dori, 4 août 2015).

Les responsables de la CRA dénoncent ce qui passe à leurs yeux pour une "tutelle paternaliste " de I'UFC sur ses associations paysannes.

\footnotetext{
${ }^{23}$ Décret $n^{\circ}$ 2001-770 bis/PRES/PM/MEF/AGRI du 31 décembre 2001 (JON¹1 2002), op.cit.
} 
Comme le montre Yves Guillermou (2003: 130) parlant du difficile dialogue à la base entre acteurs du développement rural dans l'Ouest Cameroun, cette "tutelle paternaliste » renvoie moins à une volonté hégémonique de I'UFC qu'à une stratégie pour se constituer des zones d'influence privilégiées et sauvegarder des associations paysannes qui font son identité et sa raison d'être.

En effet, la mise en place des " groupements agricoles " à l'étiquette "UFC ${ }^{24}$ a débuté en 1974. Ils remplissent les fonctions d'unités économiques et de structures de promotion du dialogue interreligieux dans les localités où ils sont implantés. De l'avis de François Ramdé ${ }^{25}$, les coopératives agricoles de I'UFC apparaissent comme "des UFC en miniature ", le reflet de la bonne cohabitation et de la valorisation de la différence à travers les villages du Séno et du Yagha. Le dialogue interreligieux acquiert de l'importance et de la visibilité à travers ces groupements composés essentiellement de catholiques et de musulmans. L'UFC possède à ce jour plus de 32 coopératives agricoles pour toutes activités confondues (maraîchage, embouche, banque de céréales, autres activités génératrices de revenus) (Ramdé, 2018).

Les agents de la DRAHSA quant à eux s'insurgent contre leur faible implication dans les études et le suivi techniques des micro-barrages de I'UFC en vertu de leur compétence reconnue dans ce domaine. Un agent technique a expliqué que la collaboration des associations et ONG avec la DRAHSA se passe sur la base d'un protocole que celles-ci signent avec I'État. Les acteurs de I'UFC feignent d'ignorer cette étape et préfèrent solliciter la DRAHSA pour des interventions ponctuelles, ou pour les besoins de reconnaissance comme ils aiment à le faire lors des Journées du maraîcher du Sahel (JMS) ${ }^{26}$ :

${ }^{24}$ Amadou Gourovo Sidibe et André Marty, 1980, op.cit.

${ }^{25}$ Actuel gestionnaire de I'UFC-Dori, Dori, 26 février 2015.

${ }^{26} C^{\prime}$ est en 2006 que l'UFC a initié les JMS au profit des groupements qu'elle encadre. Elles sont organisées chaque année entre le mois de février et mars à Dori. Pendant 72 heures les maraîchers viennent y exposer leur production. Les JMS sont non seulement un cadre d'exposition-vente, donc une opportunité de commercialisation et de visibilité sociale du projet, mais aussi un cadre d'échanges d'expériences entre producteurs. 
"Ils ont besoin de notre présence lors de ces journées comme à certains moments du processus de réalisation de leurs projets pour donner plus de crédit à leurs actions. Notre présence est une sorte de certificat pour ce qu'ils font. Mais au fond c'est quoi notre participation à ces JMS? C'est seulement une demande qu'ils adressent à la DRAHSA pour mettre à leur disposition un agent qui va les accompagner en tant que membre du jury pour la désignation du meilleur groupement de producteurs. Imaginez que le ministre et sa direction régionale, le gouverneur de la région, le hautcommissaire de la province participent à une telle cérémonie, et qu'on la rende publique avec les photos, les discours des autorités, quel bailleur refuserait d'accompagner une telle structure? Elle est la mieux organisée des ONG, elle dispose des moyens pour mobiliser tout un monde, on voit des religieux engagés pour le développement » (Dori, 6 août 2015).

La caution de l'administration territoriale dont bénéficie I'UFC semble contraindre les agents de la DRAHSA et de la CRA à composer avec elle. Ils voient cette caution comme une bienveillance a priori de l'État à l'égard de I'UFC aux dépens des autres structures, ce qui permet à ses acteurs de développer des pratiques hors normes et de se soustraire à leur encadrement. À la CRA, on admet difficilement ce traitement de faveur des autorités administratives, surtout que l'article 15 de la loi $n^{\circ} 014$ indique que "les autorités publiques veilleront au respect des lois et règlements relatifs aux sociétés coopératives et aux groupements ».

C'est ensuite sur des questions de compétence et de normes que se fondent les critiques des agents de la DRAHSA. Selon un chef de service, les réalisations hydrauliques de I'UFC ne respectent pas les normes techniques. Ses agents n'ont pas les compétences techniques pour réaliser des ouvrages de qualité. II s'étonne donc que I'ONG soit un partenaire privilégié de l'État et des bailleurs de fonds :

"On a l'impression que la tendance est à ne rien leur refuser quand ils demandent. Généralement, la plupart de ceux qui financent ne cherchent même pas à savoir comment les réalisations seront faites. Ils attendent des réalisations concrètes : des boulis construits, des cultures maraîchères qui sortent des abords des boulis, des producteurs heureux. Mais comment l'ouvrage va se faire, avec qui, pour durer combien d'années ? Est-ce que les bénéficiaires pourront prendre en charge l'ouvrage quand le projet va se retirer? Ces 
questions qui intéressent un technicien semblent ne pas avoir d'importance pour ceux qui financent les réalisations hydrauliques et agricoles de l'UFC » (Dori, 6 août 2015).

Même si les agents de la CRA et de la DRAHSA évoquent avec raison une bienveillance a priori de l'État à l'égard de l'UFC aux dépens des autres associations et ONG locales, et restent en désaccord avec l'administration de tutelle sur son traitement différencié des ONG, ils n'expriment ni officiellement, ni ouvertement leurs mésententes avec les acteurs de I'UFC. Christelle Dabiré, la chef du département des unités de production ${ }^{27}$, soutient que les interactions entre l'UFC et les deux services techniques de l'État sont bonnes. Leurs agents entretiennent des contacts réguliers, échangent sur les expériences et les opportunités. Les relations sont bonnes puisque I'UFC a toujours obtenu leur accompagnement pour certaines de ses activités.

Les remontrances viennent de responsables qui semblent défendre leurs intérêts individuels plutôt que ceux de leur service. Les critiques ne sont donc pas fondées, car les services techniques étatiques n'ont pas non plus d'exigence de qualité et de pérennité. Les raisons des critiques se situent dans le contrôle des groupements. L'UFC s'impose comme un acteur permanent d'appui et de tutelle des groupements, en concurrence avec la CRA et la DRAHSA. Cet enjeu de contrôle se double d'un enjeu financier. L'UFC mobilise directement en interne les ressources dont elle dispose pour l'appui aux groupements. Elle finance ses équipes techniques. Or, les structures publiques sont en recherche de financements, pour exercer leur rôle de tutelle de contrôle et d'appui, qu'elles n'ont pas les moyens de faire avec leurs dotations publiques, et aussi pour des compléments de revenus des agents. Elles cherchent donc à être prestataires de services pour les projets et les ONG, de façon normale et légale pour la CRA, en pratique pour la DRAHSA. Or, le fait que I'UFC joue ce rôle en direct implique qu'elle n'a que peu d'argent pour les services techniques. Leurs agents désapprouvent le fait que l'UFC se retrouve à fonctionner comme une entreprise et empiète sur « leurs compétences ».

${ }^{27}$ Dori, 28 février 2015. 
Si François Remdé refuse de considérer l'UFC comme une entreprise économique, il ne réfute pas l'idée de rechercher du profit. II souligne que la salarisation du personnel de l'ONG imposée en 1999, la prise en charge de l'important effectif non salarié des délégués des communautés, les contraintes de fonctionnement et de maintenance de la logistique, la conduisent vers l'irrésistible quête du marché. C'est pour cette raison que I'UFC exporte son expertise hors de ses zones d'intervention (Dori, 2 août 2015).

\section{Conclusion}

L'Union fraternelle des croyants (UFC) créée en 1973 lors de la grave sécheresse qu'a connue le Burkina Faso joue depuis ce temps un rôle central dans le développement rural dans la région de Dori, autour d'une double originalité : d'une part, cette ONG est codirigée par des représentants musulmans et catholiques et promeut le dialogue interreligieux, d'autre part, les financements et les appuis dont elle a bénéficié depuis cette date en ont fait la principale organisation locale de développement agricole et rural agissant dans la zone. Ses stratégies et ses rapports avec l'État se sont recomposés au terme de deux bifurcations.

La première est celle de la lutte contre les effets durables de la sécheresse, qui entraîne dès 1974 une collaboration entre l'UFC et l'État. L'UFC aligne ses interventions dans les priorités nationales et devient, dans les années 1970 et 1980, un acteur central dans le développement rural au sein des organismes permanents de l'État, l'Office national des céréales (OFNACER) et l'Organisme régional de développement (ORD). Durant cette période, ses interventions étaient appréciées par les pouvoirs publics.

Le deuxième tournant intervient au début des années 2000, où les orientations nationales sur la sécurité alimentaire et le développement rural imposent aux associations et ONG de se soumettre au contrôle et au suivi des autorités administratives et des services techniques de l'agriculture dans les régions. En continuant à agir directement et à apporter dans la durée un appui aux groupements issus de son action, I'UFC se positionne partiellement en marge de cette politique. Cela suscite des critiques fortes de la part des services techniques, qui critiquent 
également le soutien et la bienveillance que l'administration territoriale accorde à l'UFC.

Les tensions entre I'UFC et les services techniques (chambre régionale d'agriculture et direction régionale de l'agriculture) tiennent aux différences de stratégies et de positionnements, et au fait que les ressources mobilisées par l'UFC lui permettent d'agir pour partie en marge de la politique nationale. Mais les critiques des agents de la CRA et de la DRAHSA s'expliquent aussi par le fait que, faute de ressources publiques structurelles suffisantes, ces organisations sont elles-mêmes amenées à vendre leurs services. Devenus eux-mêmes des courtiers locaux, ils ne peuvent qu'entrer en concurrence avec les acteurs privés sur le terrain du développement.

Les tensions qui opposent les acteurs de l'UFC aux structures étatiques ne sont pas un cas isolé. Elles sont le reflet des évolutions qui affectent les relations entre ONG et structures publiques depuis le retour de l'État sur le devant de la scène du développement au début des années 2000.

\section{Bibliographie}

BARBEE E.-L., 1987, "Tensions in the Brokerage role. Nurses in Botswana ", Western Journal of Nursing Research, 9(2) : 244-256.

BLUNDO G., 1995, "Les courtiers du développement en milieu rural sénégalais », Cahiers d'études africaines, 137 : 73-99.

DIAWARA M., 1999, "Réseaux confessionnels de développement, pouvoirs locaux et décentralisation. Esquisse d'un modèle d'interprétation générale ", in OTAYEK (éd.), Dieu dans la cité, Tallence, CEAN : 91-107.

ENÉE G., 2010, «Les ONG au Burkina Faso: Une référence dans le champ du développement en Afrique subsaharienne ? ", Eso-Caen, 30 : 43-54.

FREYSS J., 2004, "La solidarité internationale, une profession ? Ambivalence et ambiguïtés de la professionnalisation », Revue Tiers Monde, 180 : 735772 .

GADO A.-B., 1993, Une histoire des famines au Sahel. Étude des grandes crises alimentaires ( $X I X^{e}-X X^{e}$ siècle), Paris, L'Harmattan. 
GUIGNARD J., 1996, Le Sahel de la sécheresse : septembre 1972 à septembre 1974, s.l., Éd. Sol'air.

GUILLERMOU Y., 2003, "ONG et dynamiques politiques en Afrique. Le difficile dialogue à la base entre acteurs du développement rural ", in BOURDARIAS F., HOURS B. et LE PALEC A. (éds), " Les ONG : médiations politiques et globalisation », Journal des Anthropologues, 94-95: 123143.

JANIN P., 2008, " Le soleil des indépendances (alimentaires) ou la mise en scène de la lutte contre la faim au Mali et au Sénégal », Hérodote, 131 : 92-117.

KAAG M. et SAINT-LARY M., 2011, "Nouvelles visibilités de la religion dans l'arène du développement. L'implication des élites chrétiennes et musulmanes dans les politiques publiques en Afrique ", Bulletin de l'APAD, 33 : 7-21.

KABORÉ K., 2016, «Les relations interreligieuses institutionnalisées au Burkina Faso. Le cas de I'Union fraternelle des croyants (UFC) dans le Sahel de 1960 à 2006 ", thèse de doctorat, Ouagadougou, université de Ouagadougou, $413 \mathrm{p}$.

KABORÉ K., 2017, « Mouvement interreligieux et usages d'Internet au Burkina Faso. Le cas de l'Union fraternelle des croyants (UFC) de Dori ", Émulations 24 : 23-35.

LANGEWIESCHE K., 2011, « Le dialogue interreligieux au service du développement. Élites religieuses et santé publique au Burkina Faso ", Bulletin de l'APAD, 33 : 91-119.

LAVIGNE DELVILLE P., 2013, "Déclaration de Paris et dépendance à l'aide: éclairages nigériens ", Politique africaine, 129 : 135-155.

LEBLANC M.-N., AUDET-GOSSELIN L. et GOMEZ-PEREZ M., 2013, « Les ONG confessionnelles en Afrique de l'Ouest: un équilibre précaire entre prosélytisme et professionnalisme au Burkina Faso ", Canadian Journal of Development Studies / Revue canadienne d'études du développement, $34(2): 236-256$.

OLIVIER DE SARDAN J.-P. et BIERSCHENK T., 1993, " Les courtiers locaux du développement ", Bulletin de l'APAD, 5 : 71-76.

PIVETEAU A., 1994, ONG et développement agricole au Burkina Faso. Présence majeure et effets mineurs, Paris et Ouagadougou, Orstom et MESSRS.

POUSSART-VANIER M., 2005, " La politisation de l'aide alimentaire d'urgence au Burkina Faso ", Revue Tiers Monde, 184 : 737-760. 
PRUDHOMME C., "Mission religieuse et action humanitaire : quelle continuité ? ", Annales de Bretagne et des Pays de l'Ouest, 112(2) : 11-29.

RAMDÉ P.-F., " Communication pour la paix. L'expérience de "dialogue pratique" de I'Union fraternelle des croyants (UFC) à Dori/Burkina Faso ", communication au Symposium international sur les mécanismes endogènes de pévention, gestion et résolution des conflits, Abidjan, 28, 29, 30 novembre 2018, Chaire Unesco de I'université Félix Houphouët Boigny, $10 \mathrm{p}$.

TALLET B., 1989, " Le CNR face au monde rural : le discours à l'épreuve des faits », Politique Africaine, 33 : 39-49.

\section{ANiviv}

Koudbi Kaboré est docteur en histoire contemporaine

université Ouaga1 (Burkina Faso)

E-mail : koudbikabore@yahoo.fr 